all exacerbators received inhaled corticosteroids (ICS) compared to $38.7 \%$ of the non-exacerbators. ICS treatment in patients with an exacerbation history in the 6 months prior to study inclusion was more frequent in patients with a duration of disease $>1$ year compared to those with disease duration

In the interim-analysis of 4,123 patients that have completed the 1 st year of the observational period, $25.5 \%$ had at least one exacerbation during follow-up. In the subgroups CAT30, 22.0\% and $40.2 \%$ of the patients had at least one exacerbation, respectively. A hospital stay was required for $3.5 \%$ of the patients who experienced an exacerbation of the total cohort during 12 months follow-up compared to $4.3 \%$ in the 6 month prior to the study.

Conclusion At baseline, the prevalence of patients reporting at least one exacerbation in this large real life COPD cohort was low and seems to be unchanged during 1 year follow-up.

\section{P47 RECORDING OF HOSPITALISATIONS FOR ACUTE EXACERBATIONS OF COPD IN UK ELECTRONIC HEALTHCARE RECORDS DATABASES}

${ }^{1} \mathrm{KJ}$ Rothnie, ${ }^{2} \mathrm{H}$ Mullerova, ${ }^{3} \mathrm{R}$ Hurst, ${ }^{4} \mathrm{~L}$ Smeeth, ${ }^{5} \mathrm{~J}$ Chandan, ${ }^{6} \mathrm{~K}$ Davis, ${ }^{4} \mathrm{~S}$ Thomas, ${ }^{1} J \mathrm{~K}$ Quint. 'Respiratory Epidemiology, Occupational Medicine and Public Health, National Heart and Lung Institute, Imperial College London, London, UK; ${ }^{2}$ Respiratory Epidemiology, GlaxoSmithKline R\&D, Uxbridge, UK; ${ }^{3} U C L$ Respiratory, University College London, London, UK; ${ }^{4}$ Faculty of Epidemiology and Population Health, London School of Hygiene and Tropical Medicine, London, UK; ${ }^{5}$ Medical School, Faculty of Medical Sciences, University College London, London, UK; ${ }^{6}$ Respiratory Epidemiology, GlaxoSmithKline R\&D, Upper Providence, USA

\subsection{6/thoraxjnl-2015-207770.184}

Background The Clinical Practice Research Datalink (CPRD) is a UK database of primary care health records covering 11 million residents of England and Wales, including at least 200,000 COPD patients. We have recently validated both the recording of COPD and algorithms to identify acute exacerbations of COPD (AECOPD) treated in primary care. It is unclear if primary care records alone can be used to identify hospitalisations for AECOPD. We aimed to validate strategies for identifying hospitalisations for acute exacerbations of COPD (AECOPD) using CPRD.

Methods We identified 22,599 patients with a validated diagnosis $^{1}$ of COPD who had HES data linked to CPRD. We assessed the positive predictive value (PPV) and sensitivity of four strategies to identify hospitalisations for AECOPD using CPRD: 1) AECOPD hospitalisation code; 2) AECOPD identified using our validated algorithm; 3) generic hospitalisation code; or 4) AECOPD identified using our validated algorithm and generic hospitalisation code on the same day. We identified hospitalisations for AECOPD in HES using ICD codes, and used HES identified AECOPD hospitalisation as the reference standard. We used ICD-10 codes J44.0 and J44.1 in any position and J44.9 in first position to identify hospitalisations for AECOPD in HES. We searched primary care records over a 30 day window after a record for hospitalisation for AECOPD for recording consistent with AECOPD hospitalisation defined by the four strategies. Patients were followed up between January 2004 and July 2013. As a sensitivity analysis, we repeated the analysis using a more specific definition of hospitalisation for AECOPD (J44.0 or J44.1 in first position only).

Results 19,507 hospitalisations for AECOPD were identified based on HES during the study period. The PPV and sensitivity of the various strategies to identify hospitalisations for AECOPD from CPRD alone are presented in Table 1. Sensitivity analysis did not significantly change the results.

Abstract P47 Table 1 PPV and sensitivity of different strategies to identify hospitalizations for AECOPD using primary care records compared to HES reference standard

\begin{tabular}{|l|l|l|}
\hline Strategy & PPV $(95 \% \mathrm{Cl})$ & Sensitivity $(95 \% \mathrm{Cl})$ \\
\hline AECOPD hospitalisation code & $26.5 \%(21.5-32.2 \%)$ & $1.8 \%(1.6-2.0 \%)$ \\
\hline AECOPD code & $1.9 \%(1.8-2.1 \%)$ & $27.0 \%(26.2-27.8 \%)$ \\
\hline Generic hospitalisation code & $15.5 \%(14.5-16.6 \%)$ & $29.3 \%(28.5-30.1 \%)$ \\
\hline $\begin{array}{l}\text { AECOPD code \& generic } \\
\text { hospitalisation code }\end{array}$ & $47.5 \%(41.7-53.4 \%)$ & $15.4 \%(14.8-16.1 \%)$ \\
\hline
\end{tabular}

Conclusions Primary care electronic healthcare databases are not sufficient to accurately identify hospitalisations for AECOPD. Future studies should use HES data linked with primary care records to study AECOPD which result in hospitalisation.

Sponsored by MRC and GSK.

\section{REFERENCE}

1 Quint JK, Müllerova H, DiSantostefano RL. et al. Validation of chronic obstructive pulmonary disease recording in the Clinical Practice Research Datalink (CPRD-GOLD). BMJ Open. 2014;4:e005540

\section{P48 IDENTIFYING EXACERBATIONS USING SYMPTOMS: READING BETWEEN THE LINES}

${ }^{1} \mathrm{CG}$ Johnson, ${ }^{2}$ REK Russell, ${ }^{2} \mathrm{M}$ Bafadhel. ${ }^{1}$ University of Kent, Canterbury, UK; ${ }^{2}$ University of Oxford, Oxford, UK

\subsection{6/thoraxjnl-2015-207770.185}

Introduction Exacerbations of COPD are associated with significant morbidity and mortality; however there is no clear consensus to the definition of an exacerbation and this remains subjective. Furthermore, it has been challenging to identify an individual biomarker, be it biological or physiological to identify an exacerbation, although identification of exacerbation phenotypes improves this. Most, if not all, patients report increase in symptoms during an exacerbation, measured using the visual analogue scale, performed on a $100 \mathrm{~mm}$ line ranging from no symptoms to worst ever symptoms. However, it is unclear if there is a linear relationship with the increase in VAS symptoms and the onset of an exacerbation. In this study, we seek to mathematically model relationships with the VAS and symptoms of dyspnoea, sputum production, sputum purulence and cough in patients with COPD at stable state and during exacerbations.

Methods Patients with COPD with completed assessments of VAS during both stable state and exacerbations were studied. An exacerbation was defined according to healthcare utilisation and increased symptoms. Classifier algorithms (Waikato Environment for Knowledge Analysis software ${ }^{\circledR}$ ) were run to predict the value of an exacerbation and multiple cross validation was used to assess the predictive accuracy. The Naïve Bayes (based on conditional probability), Multi-layer Perceptron (neural networks), J48 (decision tree) and Random Forest classifier were each run to model relationships.

Results Data from 149 COPD subjects was collected, with 180 instances of an exacerbation recorded. The mean (SD) VAS $(\mathrm{mm})$ for cough, dyspnoea, sputum production and purulence at baseline was 35 (27), 47 (27), 33 (27) and 28 (25) respectively. At exacerbation there was a significant increase $(\mathrm{p}<0.001)$ for all these parameters compared to stable state (mean difference, 


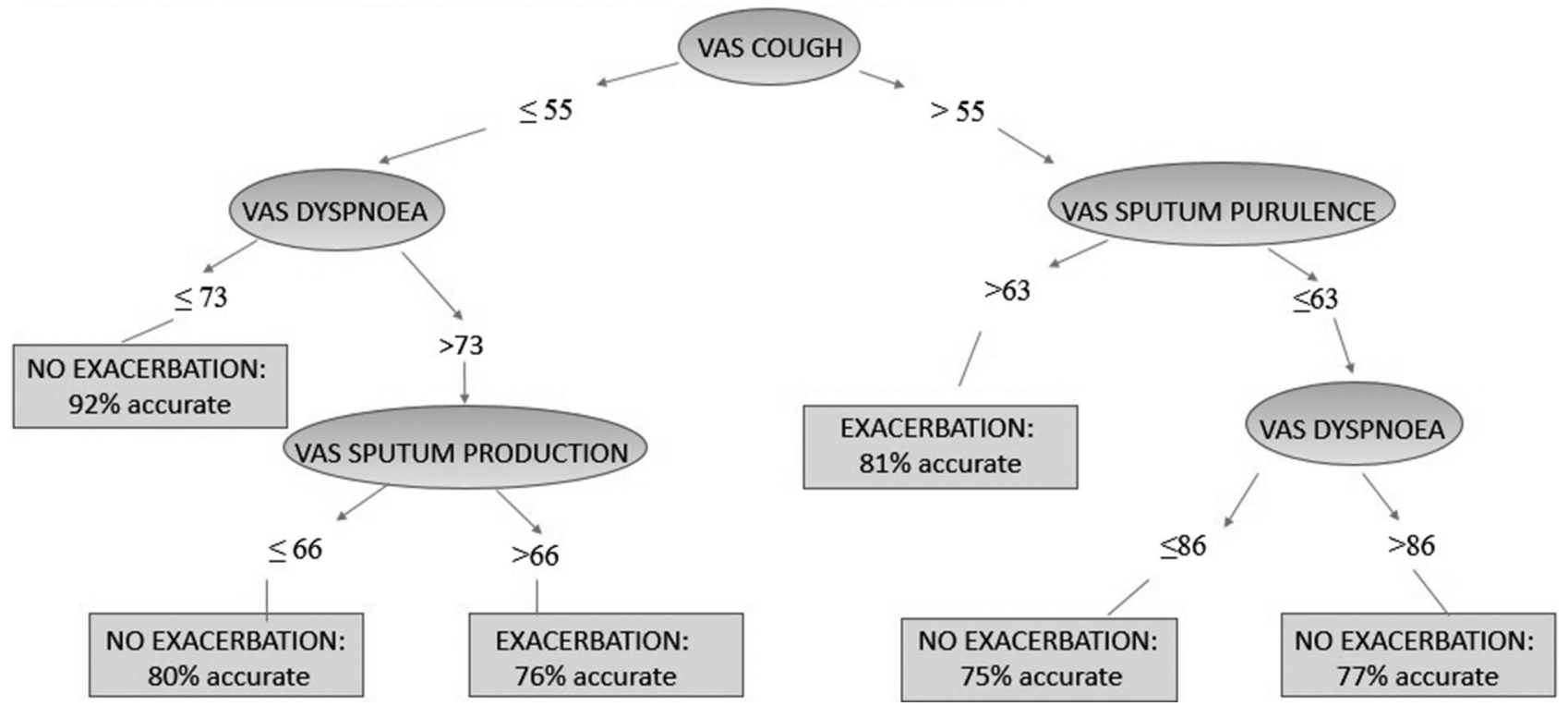

Abstract P48 Figure 1 Decision tree classfier using VAS for identification of an exacerbation of COPD

95\% CI for VAS cough, VAS dyspnoea, sputum production and purulence was $26 \mathrm{~mm}$ (20-32); $25 \mathrm{~mm}(19-30) ; 25 \mathrm{~mm}$ (19$31)$ and $25 \mathrm{~mm}(18-31)$ respectively.

The J48 classifier decision tree had the most predictive accuracy $(80 \%)$ of identifying an exacerbation (Figure 1), based on VAS and score.

Conclusion Unbiased mathematical modelling of the VAS may be useful in determining a true exacerbation event. The addition of characterisation based upon VAS may enhance the ability to identify exacerbations.

\section{P49 HIGHER SERVICE USE AMONGST PATIENTS WITH ADVANCED COPD AND PSYCHOLOGICAL CO- MORBIDITIES: ASSOCIATIONS WITH QUALITY OF LIFE, CO-MORBIDITIES AND EXACERBATIONS}

${ }^{1} \mathrm{AC}$ Gardener, ${ }^{1} \mathrm{M}$ Farquhar, ${ }^{1} \mathrm{H}$ Holt Butcher, ${ }^{1} \mathrm{C}$ Moore, ${ }^{2} \mathrm{G}$ Ewing, ${ }^{3} \mathrm{P}$ White, ${ }^{4} \mathrm{~S}$ Howson, ${ }^{5} \mathrm{R}$ Mahadeva, ${ }^{5} \mathrm{~S}$ Booth, ${ }^{6} \mathrm{P}$ Burge, ${ }^{1} \mathrm{~S}$ Mendonca. ${ }^{1}$ Primary Care Unit, University of Cambridge, Cambridge, UK; ${ }^{2}$ Centre for Family Research, University of Cambridge, Cambridge, UK; ${ }^{3}$ King's College London, London, UK; ${ }^{4}$ Cambridgeshire and Peterborough NHS Foundation Trust, UK; ${ }^{5}$ Cambridge University Hospitals NHS Foundation Trust, Cambridge, UK; ${ }^{6}$ RAND Europe, Cambridge, UK

\subsection{6/thoraxjnl-2015-207770.186}

Background There is a high prevalence of anxiety and depression among people with Chronic Obstructive Pulmonary Disease (COPD). Anxiety and depression are frequently associated with increased service use, particularly hospital admission; other demographic and health variables are also known to be significant in this relationship. However, less is known about the nature of these relationships when COPD is advanced. The aim of this study was to identify the relationship between anxiety and depression and service use in patients with advanced COPD, together with the role of key demographic and health variables.

Methods A well-characterised population-based cohort of patients with advanced COPD completed the Hospital Anxiety and Depression Scale (HADS) and reported hospital and community service use and experiences. Patient demographics and health variables were also collected. Univariate analyses of associations between service use and anxiety, depression, health variables and demographics were carried out using data collected over a three month period.

Results 235 patients recruited: mean age 71.6 (SD 10.3); 61\% male; mean MMRC dyspnea scale 3.68 (SD 1.040); mean CAT score 23.49 (SD7.5). Anxiety and depression scores (HADS) were higher than population norms. Univariate associations (Table 1) were identified between anxiety and contact with a GP $(p=0.021)$ and depression and in-patient admission $(p=$ 0.017). Other variables crudely associated with GP service use, were the number of exacerbations managed at home $(p=$ 0.006), co-morbidities $(p=0.014)$ and the CRQ dyspnea, physical and emotional domains $(p=014,0.011$ and 0.032 respectively). Crude associations were also found between inpatient admissions and the number of exacerbations at home $(p=$ $0.034)$ and the CRQ dyspnea domain $(p=0.014)$.

Abstract P49 Table 1 Univariate associations between patient factors and service use (in-patient admission and GP consultations) during a three month period

\begin{tabular}{|c|c|c|c|c|c|c|}
\hline \multirow[b]{2}{*}{$\begin{array}{l}\text { Contact in last } 3 \\
\text { months? }\end{array}$} & \multicolumn{3}{|c|}{ In-Patient admission } & \multicolumn{3}{|c|}{ Contact with GP } \\
\hline & $\mathrm{Y}$ & $\mathrm{N}$ & Sig & $Y$ & $\mathrm{~N}$ & Sig \\
\hline \multicolumn{7}{|l|}{ Anxiety $\%$} \\
\hline No/Mild & $10.2 \%$ & $89.8 \%$ & 0.104 & $65.6 \%$ & $34.4 \%$ & 0.021 \\
\hline Probable & $18.3 \%$ & $81.7 \%$ & & $81,7 \%$ & $18.3 \%$ & \\
\hline \multicolumn{7}{|l|}{ Depression $\%$} \\
\hline No/Mild & $10.5 \%$ & $89.5 \%$ & 0.017 & $68.1 \%$ & $31.9 \%$ & 0.084 \\
\hline Probable & $26.9 \%$ & $73.1 \%$ & & $84.6 \%$ & $15.4 \%$ & \\
\hline \multicolumn{7}{|c|}{ Other Variables (median and IQR) } \\
\hline $\begin{array}{l}\text { Exacerbations at home } \\
\text { (No.) }\end{array}$ & $2(0-4)$ & $1(0-2)$ & 0.034 & $2(0-4)$ & $1(0-2)$ & 0.006 \\
\hline CRQ: Dyspnea & $\begin{array}{l}3(2- \\
3.9)\end{array}$ & $\begin{array}{l}3.5(2.4- \\
4.4)\end{array}$ & 0.014 & $3(2-3.9)$ & $\begin{array}{l}3.5(2.5- \\
4.4)\end{array}$ & 0.014 \\
\hline CRQ: Physical & - & - & - & $3(2-4)$ & $\begin{array}{l}3.6(2.5- \\
4.6)\end{array}$ & 0.011 \\
\hline CRQ: Emotional & - & - & - & $\begin{array}{l}4.4(3.5- \\
5.2)\end{array}$ & $4.9(3.8-5)$ & 0.092 \\
\hline Co-morbidities & - & - & - & $4(3-6)$ & $3(2-4.8)$ & 0.012 \\
\hline
\end{tabular}

